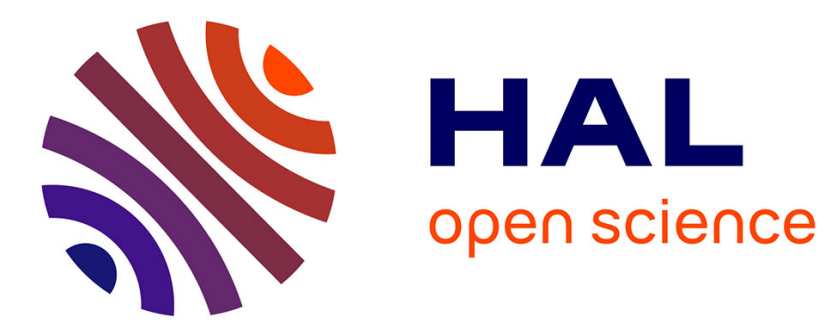

\title{
A relaxation method for large eigenvalue problems, with an application to flow stability analysis
}

\author{
Xavier Garnaud, Lutz Lesshafft, Peter Schmid, Jean-Marc Chomaz
}

\section{To cite this version:}

Xavier Garnaud, Lutz Lesshafft, Peter Schmid, Jean-Marc Chomaz. A relaxation method for large eigenvalue problems, with an application to flow stability analysis. Journal of Computational Physics, 2012, 231, pp.3912-3927. 10.1016/j.jcp.2012.01.038 . hal-00716681

\section{HAL Id: hal-00716681 \\ https://hal.science/hal-00716681}

Submitted on 11 Jul 2012

HAL is a multi-disciplinary open access archive for the deposit and dissemination of scientific research documents, whether they are published or not. The documents may come from teaching and research institutions in France or abroad, or from public or private research centers.
L'archive ouverte pluridisciplinaire HAL, est destinée au dépôt et à la diffusion de documents scientifiques de niveau recherche, publiés ou non, émanant des établissements d'enseignement et de recherche français ou étrangers, des laboratoires publics ou privés. 


\title{
A relaxation method for large eigenvalue problems, with an application to flow stability analysis
}

\author{
X. Garnaud, L. Lesshafft, P.J. Schmid, J.-M. Chomaz \\ ${ }^{a}$ Laboratoire d'Hydrodynamique, Ecole Polytechnique - CNRS, Palaiseau, France
}

\begin{abstract}
Linear stability analysis of fluid flows usually involves the numerical solution of large eigenvalue problems. We present a spectral transformation allowing the computation of the least stable eigenmodes in a prescribed frequency range, based on the filtering of the linearized equations of motion. This "shift-relax" method has the advantage of low memory requirements and is therefore suitable for large two- or three-dimensional problems. For demonstration purposes, this new method is applied to compute eigenmodes of a compressible jet.
\end{abstract}

Keywords: eigenvalue solver, spectral transformation, Krylov method, matrix-free, global modes

\section{Introduction}

The stability of fluid flow is a fundamental question in fluid dynamics, which has significant implications on the design, operation and control of flow devices. Consequently, hydrodynamic stability theory has taken a central role in fluid dynamics research, and remarkable progress has been made over the past decades. Early investigations of generic flow configurations, such as channel flows or boundary layers, have recently given way to more complex two- and three-dimensional geometries and more complex flow physics. The resulting eigenvalue problems from simple configurations were sufficiently small to allow their solution by direct techniques (such as the QR-algorithm). For more complex stability problems, however, direct techniques no longer provide a feasible solution; iterative eigenvalue algorithms have to be employed to compute a subset of the full spectrum that effectively describes the essential dynamics of small disturbances superposed on a steady base flow. Many of these algorithms for large-scale eigenvalue problems have been developed within the linear-algebra community and are readily available through several public domain libraries [12]. Among them, the two most commonly used in fluid-flow problems are the Arnoldi [16] and the Krylov-Schur [23] algorithms. These methods can, in principle, extract any portion of the full spectrum through the repeated application of a restarting step. In practice, however, only the dominant modes (i.e. those associated with the eigenvalues of largest magnitude) can be computed in many applications; yet, these modes may not provide relevant information about the physics of the problem and, in some cases, may even be spurious. As far as the asymptotic stability behavior is concerned, the least stable modes (i.e., the modes with the largest exponential growth rate) are far more important. They can be computed by coupling an iterative eigenvalue algorithm to a time-stepping routine for the linearized equations of motion (abbreviated by the linear operator $L$ ) over a given time interval $\Delta t[10]$. This way, the iterative eigenvalue solver will efficiently extract the modes that are most amplified over a time interval $\Delta t$, that is to say, it extracts the least stable modes of $L$. The propagation time $\Delta t$, in general, affects the speed of convergence of the iterative solver, but does not influence which modes the solver will converge to.

This technique is generally sufficient for bounded flows that are governed by a limited number of (or even a single) dominant instability mechanism, since the associated spectrum consists of eigenvalues that are well separated. In this case, the principal eigenvalues are easy to isolate by the iterative algorithm. When multiple and competing mechanisms are at play, the spectrum is far more complicated, and physically relevant modes are more difficult to extract. In particular, eigenvalue clusters near the neutral axis, stemming from 
continuous branches or even numerical artifacts, pose a great challenge to the convergence of the iterative algorithm. Even though unstable modes may still be extracted, the stable part of the spectrum quickly moves beyond the reach of the iterative algorithm; in this case, a different strategy is called for.

The region of convergence may be manipulated and adjusted by a rational transformation of the complex eigenvalue plane. The "shift-invert" method [16] allows the computation of the modes whose eigenvalues are closest to a complex shift parameter $\sigma$. But at each iteration of the eigenvalue solver, the method requires the solution of a linear system of the form $(L-\sigma \mathrm{I}) x=b$. Most studies accomplish the latter solution using a direct LU decomposition which has to be performed once at the start and is used for all successive iterations, until the shift parameter $\sigma$ is changed to access different parts of the spectrum. A variant of the shift-invert method, known as the Cayley transformation, yields better convergence, if an iterative solution of the linear system is chosen [18].

The LU decomposition is based on a matrix representation of the linear stability operator. Some global stability investigations used spectral discretization methods which resulted in a dense matrix of moderate size [2]. Later studies took advantage of a sparse representation, in particular, when the operator arises from a finite-element or finite-difference discretization [5]. In this case, the number of non-zero elements is proportional to the number of degrees of freedom $N$, making a sparse matrix representation convenient to handle computationally. Highly efficient multi-frontal LU solvers for large-scale sparse matrices are readily available (see e.g. $[9,3,4]$ ), but the sparsity of the output matrices is not always guaranteed. Even though the bandwidth of the factorization is not greater than that of the original matrix [11, p.152], all elements between the upper and lower band may be non-zero. Computing and storing the decomposition thus results in substantial memory requirements. For example, for the discretization of a two-dimensional problem with $N$ degrees of freedom on a structured mesh, the bandwidth scales with $N^{1 / 2}$, in which case the LU decomposition would contain up to $\mathcal{O}\left(N^{3 / 2}\right)$ non-zero elements. In three dimensions, as the bandwidth increases to $N^{2 / 3}$, the memory requirements go up as $N^{5 / 3}$. For a discretization of the compressible NavierStokes equations on a two-dimensional domain with $256 \times 512$ points using a finite-differences scheme with a six-points stencil, one can estimate that storing the LU decomposition requires about 80 GB of memory (work space requirements during the decomposition tend to be even larger), thus illustrating the limitation of this method for larger-scale problems. In some cases, appropriate reordering of the matrix entries can somewhat alleviate the problem by improving the sparsity of the factorization; this approach, however, does not provide a viable and extendable solution for large-scale problems.

An alternative that avoids the computation of the LU decomposition of the operator $L$ consists of iterative algorithms [24] to solve the linear system arising from the shift-invert or Cayley transformation. Together with ILU-type preconditioners, this approach has been applied to incompressible [15] and compressible flows [18]; in [22], the authors use un-preconditioned iterative solvers for the computation of unstable modes in plasma flows. These methods yield a reduction of computational costs associated with the solution of the linear system, but they do not provide the same level of versatility as direct methods do. Indeed, if one chooses the shift parameter $\sigma$ close to the spectrum of $L$, then $(L-\sigma I d)$ becomes ill-conditioned. In this case, the cost of preconditioning as well as the number of iterations for the linear solver to converge have to be assessed critically. In contrast, if $\sigma$ is selected farther from the spectrum, the iterative linear solver is more likely to converge with a "cheap" preconditioner, but at the same time the focusing effect of the shift-invert transformation is rather weak; consequently, it may not be possible to extract the desired modes.

The present paper presents a method for selectively extracting modal information from a linear operator $L$ without relying on the (iterative or direct) solution of a linear system. This approach has been inspired both by the "shift-invert" technique for the solution of eigenvalue problems [16] and by the selective frequency damping method of [1] for the computation of unstable steady flow. Similar to the latter method, we propose to use a relaxation procedure to selectively stabilize parts of the spectrum away from a chosen frequency shift, after which a standard Krylov method is employed to obtain the least stable modes of the relaxed system. Although the spectral transformation involved in the present "shift-relax" technique is somewhat less flexible than the "shift-invert" technique, its low memory requirement and ease of implementation make it suitable and attractive for large-scale stability computations of two- or three-dimensional flows. 


\section{Description of the method}

\subsection{Definition of the problem}

Let the dynamics of the problem under consideration be governed by a set of non-linear equations of the form

$$
\dot{\boldsymbol{q}}=F(\boldsymbol{q}),
$$

where $\boldsymbol{q}$ is the state vector and $F$ denotes a discrete integro-differential operator with appropriate boundary conditions. For simplicity, only finite-dimensional operators (which arise after spatial discretization) will be considered in this paper. We assume that this operator has a fixed point $\boldsymbol{q}_{0}$, such that $F\left(\boldsymbol{q}_{0}\right)=0$. If this base state is stable to finite-amplitude perturbations, it can be computed by integrating the dynamical system (1) over a sufficiently long time. Algorithms such as the Newton-Krylov method [10] or Selective Frequency Damping (SFD) [1] can be used to obtain a base state even in unstable situations. The SFD method relies on low-pass filtering of the equations of motion in order to suppress high-frequency instabilities. The present method generalizes this approach in order to compute modes of a linear operator in a selected frequency band.

Let $L=\nabla_{\boldsymbol{q}} F\left(\boldsymbol{q}_{0}\right)$ be the linearization of $F$ about the steady base state $\boldsymbol{q}_{0}$. Based on the decomposition $\boldsymbol{q}=\boldsymbol{q}_{0}+\boldsymbol{q}^{\prime}$, the dynamics of small perturbations $\boldsymbol{q}^{\prime}$ is governed by the linear system

$$
\dot{\boldsymbol{q}}^{\prime}=L \boldsymbol{q}^{\prime} .
$$

Temporal modes of (2) are sought in the form

$$
\boldsymbol{q}^{\prime}(\boldsymbol{x} ; t)=\widetilde{\boldsymbol{q}}(\boldsymbol{x}) \exp (-\mathrm{i} \omega t),
$$

such that the spatial structure $\widetilde{\boldsymbol{q}}$ satisfies

$$
-i \omega \widetilde{\boldsymbol{q}}=L \widetilde{\boldsymbol{q}} .
$$

A complex eigenfrequency $\omega$ is associated with the eigenmode $\widetilde{\boldsymbol{q}}$.

If an iterative solver is used to compute the least stable modes of (3) using an iterative eigenvalue solver, it is of advantage to consider the operator that takes a state $\boldsymbol{q}^{\prime}(0)$ as an initial condition for (2) and returns the state $\boldsymbol{q}^{\prime}(\Delta t)$ after a given time $\Delta t$. This operator is referred to as the propagator and can be formally written as

$$
P=\exp (\Delta t L) .
$$

Krylov-based iterative eigenvalue solvers identify a subset of the eigenvalues $\lambda=\lambda_{r}+\mathrm{i} \lambda_{i}$ with the largest absolute value $|\lambda|$ (in the following, the subscript $r$ and ${ }_{i}$ respectively denote the real and imaginary parts of a complex number). Modes of $P$ with the largest $|\lambda|$ are identical to modes of $L$ with the largest growth rate $\omega_{i}$. Even though the explicit computation of the matrix $P$ would be an onerous task, its application to vectors can be easily performed by a standard time marching method; this makes the use of $P$ appropriate for an iterative solver.

For some flows however, it may be interesting to investigate modes that belong to different frequency ranges, as these may correspond to different physical mechanisms (see for example the case of the flow around a leading edge [19] or that of supersonic jet flows [20]). In this case the objective is to compute the least stable modes with a real frequency $\omega_{r}$ close to a given value $\omega_{0}$. The present method achieves this goal by applying a bandpass temporal filter to the propagator, such that modes with real frequency $\omega_{r}$ far from the target frequency $\omega_{0, r}$ are attenuated.

\subsection{Filtering}

In this section we recall standard filtering results applied to a signal $\boldsymbol{y}(t)$ with $t \in \mathbb{R}$. Its Fourier transform reads

$$
\boldsymbol{y}(t)=\int_{-\infty}^{\infty} \hat{\boldsymbol{y}}(\omega) \exp (-\mathrm{i} \omega t) \mathrm{d} \omega .
$$


We proceed by damping out the components of $\boldsymbol{y}(t)$ with frequencies far from a given value $\omega_{0}$ using a standard first-order bandpass filter whose transfer function is given by

$$
H(\omega)=\frac{1}{1-i \frac{\omega-\omega_{0}}{\tau}} .
$$

The filtered signal $\overline{\boldsymbol{y}}(t)$ is then given by the convolution

$$
\overline{\boldsymbol{y}}(t)=\int_{-\infty}^{\infty} H(\omega) \hat{\boldsymbol{y}}(\omega) \exp (-\mathrm{i} \omega t) \mathrm{d} \omega,
$$

which satisfies the filtered ordinary differential equation

$$
\dot{\overline{\boldsymbol{y}}}=-\mathrm{i} \omega_{0} \overline{\boldsymbol{y}}-\tau(\overline{\boldsymbol{y}}-\boldsymbol{y}) .
$$

The above bandpass filter is centered about the target frequency $\omega_{0}$ and has a half width at half maximum of $\sqrt{3} \tau$.

The presented analysis applies to signals defined for all times. If one wants to use the differential equation (5) to filter a signal defined only for $t \geq 0$, initial conditions have to be specified. The overall frequency selection effect, however, will prevail independent of initial conditions, if the differential equations have been sufficiently advanced in time. In this way, the differential equation (5) provides a way to filter a signal without having to store its entire history.

\subsection{Selective frequency damping of a linear dynamical system}

Following [1], let us consider the following linear dynamical system:

$$
\begin{aligned}
& \dot{\boldsymbol{q}}^{\prime}=L \boldsymbol{q}^{\prime}-\chi\left(\boldsymbol{q}^{\prime}-\overline{\boldsymbol{q}}^{\prime}\right), \\
& \dot{\overline{\boldsymbol{q}}}^{\prime}=-\mathrm{i} \omega_{0} \overline{\boldsymbol{q}}^{\prime}-\tau\left(\overline{\boldsymbol{q}}^{\prime}-\boldsymbol{q}^{\prime}\right) .
\end{aligned}
$$

Equation (6b) represents a differential equation corresponding to the bandpass filter introduced above. The signal $\overline{\boldsymbol{q}}^{\prime}$ is therefore a filtered version of $\boldsymbol{q}^{\prime}$, in which frequency components far from the target frequency $\omega_{0}$ are damped.

The right-hand side of (6a) is a sum of two terms. The first one represents the linear operator, while the second part acts as a proportional controller that drives the variable $\boldsymbol{q}$ towards its filtered counterpart $\overline{\boldsymbol{q}}$.

The SFD procedure of [1] applies the same filtering with $\omega_{0}=0$ to a non-linear operator instead of $L$ in (6). In this case, any fixed point of $F$ corresponds to a fixed point of the extended system, the filtered state $\overline{\boldsymbol{q}}^{\prime}$ being then equal to the full state. However, the stability of the fixed point of the extended system is modified and trajectories differ. Our shift-relax (SR) approach relies on the observation that the frequency selection procedure not only preserves the fixed points of non-linear operators, but also the eigenmodes of linear systems, as will be demonstrated next.

In order to study the relationship between the spectra associated with the original linear dynamical system (2) and the SR extension (6), we introduce the composite filtered operator $\mathcal{F}$ defined as

$$
\mathcal{F} \equiv\left(\begin{array}{c|c}
L-\chi \mathrm{Id} & \chi \mathrm{Id} \\
\hline \tau \mathrm{Id} & \left(-\mathrm{i} \omega_{0}-\tau\right) \operatorname{Id}
\end{array}\right)
$$

such that (6) can be rewritten as

$$
\left(\begin{array}{c}
\dot{\boldsymbol{q}}^{\prime} \\
\dot{\overline{\boldsymbol{q}}}^{\prime}
\end{array}\right)=\mathcal{F}\left(\begin{array}{c}
\boldsymbol{q}^{\prime} \\
\overline{\boldsymbol{q}}^{\prime}
\end{array}\right) .
$$

If the state vector $\boldsymbol{q}^{\prime}$ contains $N$ elements, then a $2 N$-dimensional eigenvector of $\mathcal{F}$ associated with the complex eigen-frequency $\Omega$, i.e.

$$
\mathcal{F} \widetilde{\boldsymbol{Q}}=-\mathrm{i} \Omega \widetilde{\boldsymbol{Q}},
$$


can be decomposed into two $N$-dimensional components, $\widetilde{\boldsymbol{Q}}=(\widetilde{\boldsymbol{q}}, \widetilde{\boldsymbol{q}})^{T}$. According to (6b), these two components are related by

$$
\widetilde{\boldsymbol{q}}=\frac{1}{1-\mathrm{i} \frac{\Omega-\omega_{0}}{\tau}} \widetilde{\boldsymbol{q}} .
$$

Consistent with the design of the filter, the scalar factor between $\widetilde{\boldsymbol{q}}$ and $\widetilde{\boldsymbol{q}}$ can be related to the transfer function (4). We can substitute this result back into (6a) to obtain

$$
L \widetilde{\boldsymbol{q}}=-\mathrm{i} \omega(\Omega) \widetilde{\boldsymbol{q}}
$$

with

$$
\omega(\Omega)=\Omega+\mathrm{i} \chi\left(1-\frac{1}{1-\mathrm{i} \frac{\Omega-\omega_{0}}{\tau}}\right) .
$$

Equation (8) shows that $\widetilde{\boldsymbol{q}}$, i.e. the vector corresponding to the first N elements of $\widetilde{\boldsymbol{Q}}$, is an eigenvector of $L$. This justifies the use of the SR system (6) as a spectral transformation, as the modes of $L$ can be recovered from those of $\mathcal{F}$.

Equation (9) characterizes the mapping between the spectra of $L$ and $\mathcal{F}$. For any eigenvector $\widetilde{\boldsymbol{Q}}=(\widetilde{\boldsymbol{q}}, \widetilde{\overline{\boldsymbol{q}}})^{T}$ of $\mathcal{F}$ with complex frequency $\Omega, \widetilde{\boldsymbol{q}}$ is an eigenvector of $L$ with complex frequency $\omega(\Omega)$ given by (9). This latter equation can be re-arranged as a second-order polynomial in $\Omega$ where $\omega$ appears as a parameter. This shows that two values of $\Omega$ correspond to a single value of $\omega$, which is consistent with the fact that the dimension of $\mathcal{F}$ is twice the dimension of $L$.

Proceeding with the analysis of the mapping between the spectra of the original operator $L$ and its SR extension $\mathcal{F}$, let us introduce the following scaled variables

$$
\left(\omega^{\prime}, \Omega^{\prime}, \chi^{\prime}\right)=\frac{1}{\tau}\left(\omega-\omega_{0}, \Omega-\omega_{0}, \chi\right) .
$$

Equation (9) can then be simplified as follows

$$
\omega^{\prime}=\Omega^{\prime}+\mathrm{i} \chi^{\prime}\left(1-\frac{1}{1-\mathrm{i} \Omega^{\prime}}\right) .
$$

It therefore appears that after a shift of origin (given by the parameter $\omega_{0}$ ) and a scaling (given by the factor $\tau$ ) of the complex-frequency plane, the transformation can be studied in terms of one single parameter $\chi^{\prime}=\chi / \tau$.

Effect of $\chi^{\prime}$. The parameter $\chi^{\prime}$ measures the gain of the proportional controller relative to the frequency scale $\tau$. The analysis (given in detail in Appendix A) shows how $\chi^{\prime}$ influences the spectral transformation. We observe that the transformation is self-similar with respect to two transformations of $\omega^{\prime}$ and $\Omega^{\prime}$ : first, by different shifts of origins of these two variables and, second, by a scaling by the factor $\chi^{\prime-1 / 2}$. The shift of origin for $\Omega^{\prime}$ has no influence on the frequency selection effect of the filter, as it changes neither the order in which modes will be extracted nor the separation between the eigenvalues of the filtered propagator. The scaling is more relevant, but its effect on the transformation is the same as that of $\tau$. Acting on $\chi^{\prime}$ therefore adds no additional flexibility to the method. In an effort to keep the scaling of the complex plane and the shift of origin as two parameters, we choose $\chi^{\prime}=1$ throughout our study and only use $\omega_{0}$ and $\tau$ as changing parameters.

Study of the transformation for $\chi^{\prime}=1$. For a given value of $\omega^{\prime},(10)$ is a second-order polynomial in $\Omega^{\prime}$. As mentioned previously, this results in two solution branches, for which an analytic expression can easily be obtained. We note that the distinction between the two branches is not unique, as it depends on the location of the branch cut for the square root function with complex arguments. In the following representation we chose the common branch cut for negative real arguments of the square root. 
Figure $1 a, b$ illustrates the mapping between the original spectral plane $\omega^{\prime}$ and the two associated values of $\Omega_{1,2}^{\prime}$ by the SR transformation, respectively indicated by blue and black lines, together with the transformation of several sample values of $\omega^{\prime}$ represented by colored symbols. These values are chosen arbitrarily for illustration purposes. The solid and dashed lines in figure $1 b$ are, respectively, the images of iso- $\omega_{i}^{\prime}-$ and iso- $\omega_{r}^{\prime}$-lines in the $\omega^{\prime}$-plane represented in figure 1a. The mapping (with the chosen branch cut) defines two regions of the complex $\Omega^{\prime}$-plane. Values of $\Omega^{\prime}$ inside the unit circle centered at $\Omega^{\prime}=-\mathrm{i}$ correspond to the first root $\Omega_{1}^{\prime}$ while values outside the circle correspond to the second root $\Omega_{2}^{\prime}$. Accordingly, the sample values represented by colored symbols in figure $1 a$ has two images in the $\Omega^{\prime}$-plane. We notice, however, that neither the mapping $\omega^{\prime} \rightarrow \Omega_{1}^{\prime}$ nor the mapping $\omega^{\prime} \rightarrow \Omega_{2}^{\prime}$ is continuous.

$(a)$

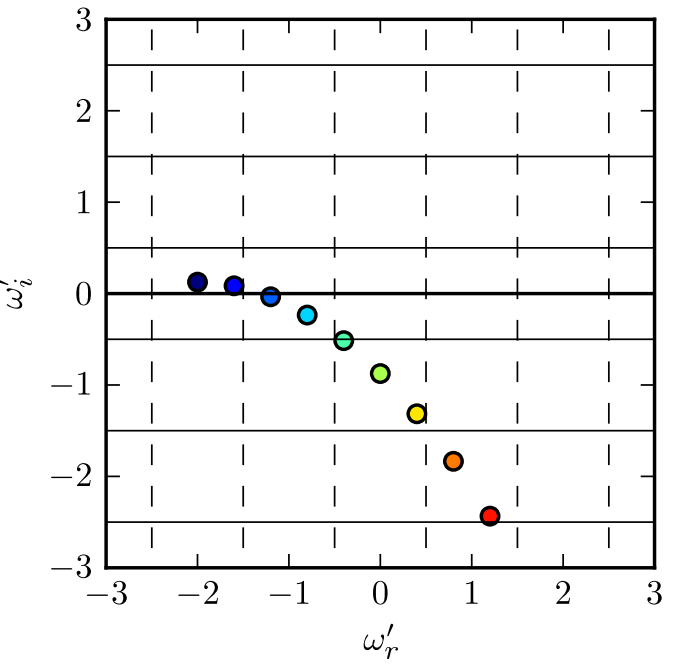

(b)

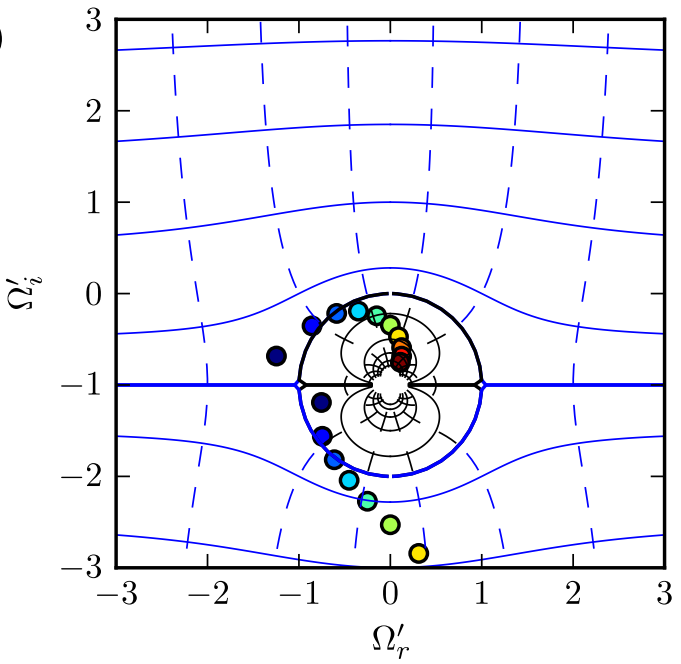

Figure 1: Mapping between the original spectral plane $\omega^{\prime}$ (a) and its SR-transformed image $\Omega^{\prime}$ (b). The colored symbols correspond to sample values.

The images of the sample values in figure $1 b$ suggest another distinction between the two images of $\omega^{\prime}$. We respectively define ${\Omega^{\prime}}^{+}$and ${\Omega^{\prime}}^{-}$as the images of $\omega^{\prime}$ such that the following relation involving the transformed growth rates is satisfied

$$
\Omega_{i}^{\prime-}\left(\omega^{\prime}\right) \leq-1 \leq{\Omega^{\prime}}_{i}^{+}\left(\omega^{\prime}\right) .
$$

See Appendix A for a proof. As $\tau$ is real and positive, the above distinction between the two branches results in

$$
\Omega_{i}^{-} \leq \omega_{0, i}-\tau \leq \Omega_{i}^{+},
$$

expressed in terms of the non-scaled variables.

The least stable modes of the SR operator $\mathcal{F}$ (given in (7)) will then be extracted by applying an eigenvalue solver to the filtered propagator

$$
\mathcal{P}=\exp (\mathrm{i} \Delta t \mathcal{F})
$$

as motivated in $\S 2.1$. This will extract the least stable modes of the filtered operator $\mathcal{F}$. Consequently, we can restrict our attention to the $\Omega^{\prime}$ branch. Furthermore, as only the growth rate rather than the frequency decides which modes will be extracted, the relevant features of the transformation can be studied in terms of the single-valued real function $\omega^{\prime} \rightarrow \Omega_{i}^{\prime}$. This function is represented in figure 2 .

Three regions of the $\omega^{\prime}$-plane can be identified:

- For $1 \lesssim \omega_{i}^{\prime}$, the transformed growth rate ${\Omega^{\prime}}_{i}^{+}$behaves similar to the un-transformed growth rate $\omega_{i}^{\prime}$. No significant dependency with $\omega_{r}^{\prime}$ is observed, indicating that no noticeable frequency selection occurs. 


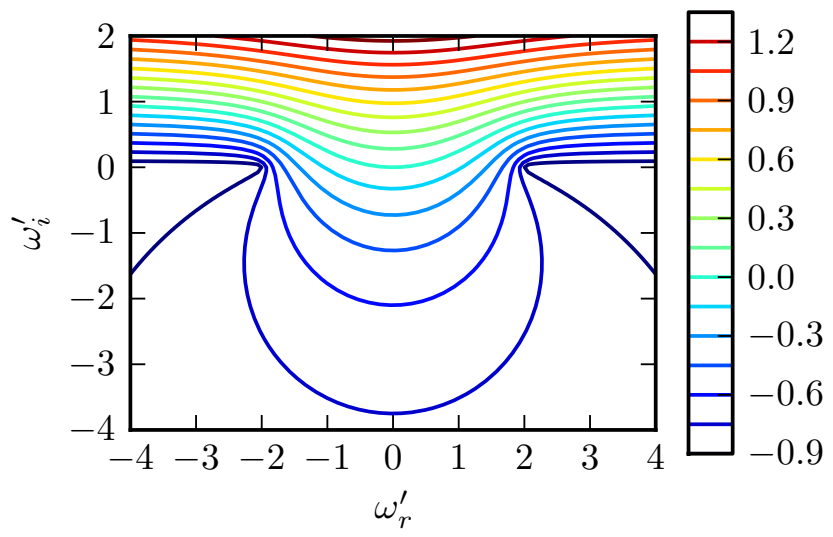

Figure 2: Imaginary part of the eigenvalue of the SR operator $\Omega_{i}^{\prime+}\left(\omega^{\prime}\right)$ as a function of the eigenvalue $\omega^{\prime}$ of the origin for $\chi^{\prime}=1$.

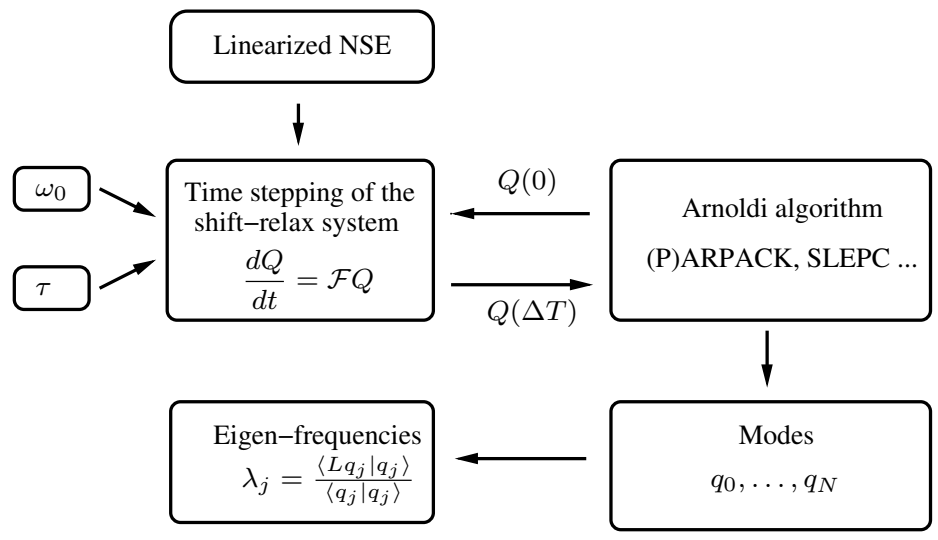

Figure 3: Practical implementation of the method

- For $-2 \lesssim \omega_{i}^{\prime} \lesssim 1$, the value of $\Omega_{i}^{\prime+}\left(\omega^{\prime}\right)$ exhibits strong dependency on the real frequency $\omega_{r}^{\prime}$. Along a line of constant $\omega_{i}$, the value of $\Omega_{i}^{\prime+}\left(\omega^{\prime}\right)$ reaches a maximum for $\omega_{r}=0$, confirming that the filter plays the expected role of stabilizing modes with real frequencies far from $\omega_{0, r}$.

- For $\omega_{i}^{\prime} \lesssim-2, \Omega_{i}^{\prime+}\left(\omega^{\prime}\right)$ is approximately constant. The dynamics of the SR system is dominated by the control term in (6a) which conceals the dynamics of the linear operator $L$.

The above representation allows to determine the behavior of the proposed method. For $\omega_{i} \gtrsim \omega_{0, i}+\tau$, the spectral transformation produces results similar to that of the propagator approach (see $\S 2.1$ and [10]). The frequency selection effect is insignificant in this region. Strong frequency selection is achieved in the range of frequencies $\omega_{0, i}-2 \tau \lesssim \omega_{i} \lesssim \omega_{0, i}+\tau$, as the effect of the bandpass filter is clearly present. Modes with real frequencies outside a bandwidth of order $\tau$ centered around $\omega_{0, r}$ are damped. In this range of growth rates, modes with real frequencies close to $\omega_{0, r}$ will be extracted first. All modes with a growth rate $\omega_{i} \lesssim \omega_{0, i}-2 \tau$ will map to essentially the same growth rate for the SR operator. These modes will be difficult to distinguish with an iterative eigenvalue solver.

\subsection{Numerical considerations}

Implementation. A practical implementation of the present method requires only few additions to a standard DNS code. These are summarized in figure 3. First, a routine evaluating the linearized Navier-Stokes operator is needed. The linearization may be carried out by hand, as done in the examples given in this paper, or numerically "on the fly" from a non-linear routine, as done in [14]. 
Next, the eigenvalue extraction is performed. Several libraries for both sequential and parallel implementations may be used for this task. The Krylov-Schur solver provided in the SLEPc library [23] is used here to extract the eigenmodes of the SR operator, with 60 Krylov vectors. Better convergence behavior has been observed compared to the Implicitly Restarted Arnoldi Method (IRAM), which is more frequently used for the computation of modes in fluid flow problems[16]. In [22], the authors report good results using the "harmonic extraction" solver of SLEPc for frequency selection. For our cases, this method has not produced the desired outcome.

Finally, only minor changes in order to integrate the filtering procedure into a standard time-stepping routine are necessary. The algorithm for the time stepping of the SR dynamical system is exemplified using an explicit Euler scheme as outlined below. The variables $q_{1}$ and $q_{2}$, respectively, represent the state and its filtered counterpart, $L$ is the linear flow operator, and $\delta t$ is a discrete time step.

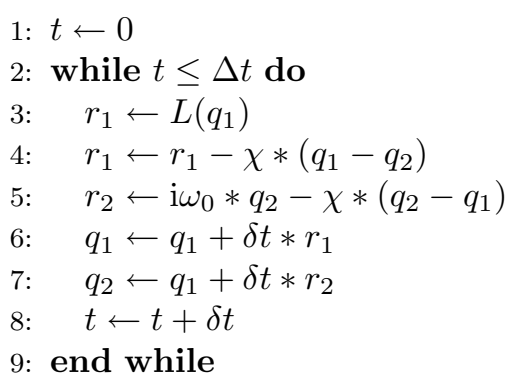

The algorithm differs from standard time stepping by the additional lines 4,5 and 7 . These correspond to a small number of operations compared to the application of the operator $L$, in particular, when high-order schemes are employed; the SR computations are therefore only slightly slower than regular time-stepping, and they can be just as efficiently parallelized.

Memory requirements. The memory requirement of the present method is twice that of a standard propagator technique, as the dimension of the phase space is doubled due to the presence of the filtered variables. If the eigenvalue solver and the time stepper respectively require $n c v$ and $n t s$ vectors of size $N$ (the number of degrees of freedom associated with the discretization of the equations) as workspace, most of the memory requirement will originate from the storage of these $2 N(n c v+n t s)$ values. For the discretization of the compressible Navier-Stokes equations mentioned in the introduction with $n c v=60$, nts $=20$ and double precision complex arithmetics, the program would require $2 \times 5 \times 256 \times 512 \times 80 \times 16 \mathrm{~B} \approx 1.6 \mathrm{~GB}$ of memory, which is substantially less than the $80 \mathrm{~GB}$ required for the sparse LU decomposition alone.

Effect of the propagation time. The effect of the propagation time on the SR operator is the same as for the classical propagator method [10]. The actual value of the propagation time $\Delta t$ should not influence which subset of modes will be extracted. The propagation time will however affects the convergence of the eigenvalue solver. If $\Delta t$ is small, only a few time marching steps are required in each iteration; nonetheless, a large number of iterations will be necessary in order to reach a desired accuracy. It will also require a large number of restarts, which ultimately may affect robustness. On the other hand, if $\Delta t$ is too large, each iteration will be rather costly since it consists of many time steps, and the method will make less use of the orthogonalization step. A balance has to be found between the computational time needed to propagate the solution forward in time, the cost associated with the eigenvalue solver and the robustness of the restarting procedure.

Time stepping accuracy. Numerical time stepping methods such as Euler and Runge-Kutta schemes correspond to an approximation of the exponential matrix propagator by a matching polynomial. Such an approximation preserves the modes: it therefore introduces no error on the computed modes. In practical applications, the time step $\delta t$ of the time-stepping routine is much smaller than the characteristic time scale of modes one wishes to compute. As a consequence, the spectrum transformation introduced by numerical time stepping will be nearly exponential in the range of eigenfrequencies of interest, such that the rules derived earlier for the choice of parameters remain valid. 
Eigenvalue recovery. As explained in $\S 2.3$, recovering the eigenvectors of $L$ is straightforward, as they correspond to the first $N$ components of the eigenvectors of the SR propagator. The eigenfrequency $\omega$ associated with an eigenvector $\boldsymbol{q}$ of $L$ may conveniently be obtained by computing the Rayleigh quotient

$$
-\mathrm{i} \omega=\frac{\langle\boldsymbol{q} \mid L \boldsymbol{q}\rangle}{\langle\boldsymbol{q} \mid \boldsymbol{q}\rangle} .
$$

Alternatively, the eigenvalue $\Omega$ of the SR operator may be computed from the eigenvalue $\lambda$ of the SR propagator via

$$
\Omega=\frac{\log (\lambda)}{-\mathrm{i} \Delta t},
$$

and (9). Method (12) has three advantages over the latter method. First, as the logarithm is multivalued, the above formula yields the imaginary part only if one knows the Riemann sheet $\Omega$ lies on, i.e. for example that $0 \leq \Omega_{r} \leq 2 \pi / \Delta t$. Second, the numerical time stepping makes (13) inexact: as discussed in $\S 2.4$, the transformation introduced by time-stepping is not exactly exponential but rather a polynomial approximation. This problem does not arise for the Rayleigh quotient (12). Finally, (12) minimizes the residual $\|L \boldsymbol{q}+\mathrm{i} \omega \boldsymbol{q}\| /\|\boldsymbol{q}\|$, the norm being that corresponding to the inner product in (12). This inner product may include weights that select specific flow quantities of regions. In all computation presented in $\S 3$ and 4 , the $l^{2}$ inner product on the state vector components is chosen as it is the one used in the Krylov-Schur algorithm.

\section{Application to the local and global stability analysis of a compressible jet}

\subsection{Governing equations for local and global computations}

We consider a compressible jet of radius $R$, with characteristic velocity $U_{0}$, density $\rho_{0}$ and temperature $T_{0}$ measured on the centerline, discharging into a fluid at rest with density $\rho_{\infty}$ and temperature $T_{\infty}$. These same quantities are used to make the problem non-dimensional. In a cylindrical coordinate system $(x, r, \theta)$, the nonlinear governing equations are expressed in terms of the conservative flow variables $\boldsymbol{q}=\left(\rho, \rho u_{x}, \rho u_{r}, \rho u_{\theta}, \rho E\right)^{T}$, where $\rho$ is the density, $\boldsymbol{u}=u_{x} \boldsymbol{e}_{x}+u_{r} \boldsymbol{e}_{r}+u_{\theta} \boldsymbol{e}_{\theta}$ is the flow velocity, and $E$ denotes the total energy [25]. The Reynolds, Mach and Prandtl numbers are defined as

$$
\operatorname{Re}=\frac{U_{0} R \rho_{0}}{\mu}, \quad \operatorname{Ma}=\frac{U_{0}}{c_{0}}, \quad \operatorname{Pr}=\frac{\mu C_{p}}{\kappa}=1,
$$

with $c_{0}$ the reference speed of sound on the jet axis, $C_{p}$ the ambient specific heat at constant pressure, $\mu$ the dynamic viscosity and $\kappa$ the thermal conductivity of the fluid. The fluid properties $C_{p}, \mu$ and $\kappa$ are assumed to be constant throughout the flow.

For the purpose of a stability analysis, the flow variables $\boldsymbol{q}$ are decomposed into a steady axisymmetric base flow $\boldsymbol{q}^{b}$ and unsteady perturbations $\boldsymbol{q}^{\prime}$, such that $\boldsymbol{q}(x, r, \theta, t)=\boldsymbol{q}^{b}(x, r)+\boldsymbol{q}^{\prime}(x, r, \theta, t)$. The governing equations are then linearized around $\boldsymbol{q}^{b}$, and a normal mode ansatz for $\boldsymbol{q}^{\prime}$ allows to characterize the temporal growth or decay of perturbation eigenmodes of the linear system. Both local (§3.2) and global (§ 3.3 and 4) normal modes will be considered in the following. Local theory assumes the flow to be infinite, parallel and uniform in the $x$ - direction. In this case, perturbations are Fourier-transformed in $x$, leaving only $r$ as an eigendirection. Global theory, by contrast, considers spatially developing base flows, and accounts for boundary conditions at the inlet and outlet. In the global framework, both $r$ and $x$ are thus eigendirections, which leads to a system size that precludes the use of direct eigenvalue solvers.

\subsection{Validation: direct computation of local temporal eigenmodes}

A validation of the present SR propagator method is conducted by computing the eigenmodes of a parallel jet of infinite streamwise extent. This test case represents the local stability problem, as it has been widely 
used to describe the stability properties of slowly varying flows [13]. The base flow is prescribed as

$$
\begin{aligned}
u_{x}^{b} & =\frac{1}{2}\left\{1+\tanh \left[2\left(r-\frac{1}{r}\right)\right]\right\}, \\
u_{r}^{b} & =u_{\theta}^{b}=0, \\
T^{b} & =S+(1-S) u_{x}^{b}+\frac{\gamma-1}{2} \mathrm{Ma}^{2} u_{x}^{b}\left(1-u_{x}^{b}\right), \\
\rho^{b} & =T^{b^{-1}},
\end{aligned}
$$

from which we obtain the total energy $\rho^{b} E^{b}=\gamma^{-1}(\gamma-1)^{-1} M a^{-2}+\rho^{b} u_{x}^{b^{2}} / 2$, with uniform pressure $p^{b}=$ $\left(\gamma \mathrm{Ma}^{2}\right)^{-1}$. The ratio of specific heats is taken as $\gamma=1.4$, and $S=T_{\infty} / T_{0}$ denotes the ambient-to-jet temperature ratio. A hot jet with $S=0.5$ is chosen for the present configuration, along with the parameters $\mathrm{Re}=500, \mathrm{Ma}=0.4$ and $\mathrm{Pr}=1$.

Azimuthal periodicity and streamwise invariance justify a normal mode ansatz of the form

$$
\boldsymbol{q}^{\prime}(x, r, \theta, t)=\widetilde{\boldsymbol{q}}(r) \exp [\mathrm{i}(k x+m \theta-\omega t)]+c c,
$$

where $c c$ stands for the complex conjugate. Within the framework of a temporal stability analysis, the complex frequency $\omega$ is sought as a function of prescribed streamwise and azimuthal wave-numbers $k \in \mathbb{R}^{+}$ and $m \in \mathbb{Z}$.

One-dimensional reference solution. Upon substitution of the base flow (15) and normal mode perturbations (16) into the linearized equations of motion, only the radial coordinate direction needs to be discretized. We use a compact finite-difference scheme [17] for the spatial discretization and impose homogeneous Dirichlet boundary conditions at $r=150$, leading to the discrete local temporal eigenvalue problem of the form

$$
\mathbf{A}(k, m) \widetilde{\boldsymbol{q}}=\omega \mathbf{B} \widetilde{\boldsymbol{q}} .
$$

All eigenmodes $(\omega, \widetilde{\boldsymbol{q}})$ are then computed via a QR-algorithm. The most relevant part of the spectrum for values $k=m=1$ is shown in figure $4 a$. Three families of eigenmodes may be distinguished based on their frequency $\omega_{r}$, their growth rate $\omega_{i}$, and their spatial distributions of azimuthal vorticity (figure $4 b$ ) and dilatation $\boldsymbol{\nabla} \cdot \boldsymbol{u}$ (figure $4 c$ ).

Vortical perturbations localized in the fluid at rest outside the jet form the classical continuous spectrum of unbounded shear flows [26] with near-zero real frequency. Due to the finite size of the numerical domain, continuous branches results in densely clustered discrete modes in the discretized problem. These are represented by blue symbols that line up close to the $\omega_{r}=0$ axis in figure $4 a$. Acoustic waves in the freestream form two continuous branches, both plotted in red in figure $4 a$. These are characterized by very slow temporal decay $\left(\omega_{i} \approx 0\right)$ and frequencies in the continuous ranges $\omega_{r} \leq-k \sqrt{S} / \mathrm{Ma}$ and $\omega_{r} \geq k \sqrt{S} / \mathrm{Ma}$ . These cut-off frequency $\pm k \sqrt{S} / \mathrm{Ma}$ corresponds to cylindrical acoustic waves traveling parallel to the jet axis at the speed of sound $c_{\infty}$ in the outer flow. Real parts of vorticity and dilatation fields are shown in figures $4 b, c$ as a function of $r$ for particular modes (vortical mode with $\omega=2.24 .10^{-6}-1.49 .10^{-3} \mathrm{i}$ in blue and acoustic mode with $\omega=2.03-1.91 .10^{-3} \mathrm{i}$ in red). A fourth branch of modes is represented by green symbols in figure $4 a$. This branch consists of discrete modes with a single unstable one marked by a cross. The spatial structure of the unstable eigenmode is plotted in green in figures $4 b, c$. This mode displays strong vorticity perturbations inside the jet shear-layer around $r=1$, and nearly no dilatation perturbation. As the discrete modes (green symbols in figure $4 a$ ) have real frequencies below the acoustic cut-off value (i.e. a subsonic phase velocity), no sound is radiated by these modes.

\subsection{Local eigenmodes via the SR method on a two-dimensional periodic domain}

In order to demonstrate the use of the SR technique, it is applied to reproduce a selected part of the spectrum shown in figure $4 a$. Local modes may be computed as global eigenmodes on a two-dimensional $(x, r)$ domain,

$$
\boldsymbol{q}^{\prime}(x, r, \theta, t)=\widetilde{\boldsymbol{q}}(x, r) \exp [\mathrm{i}(m \theta-\omega t)],
$$


(a)
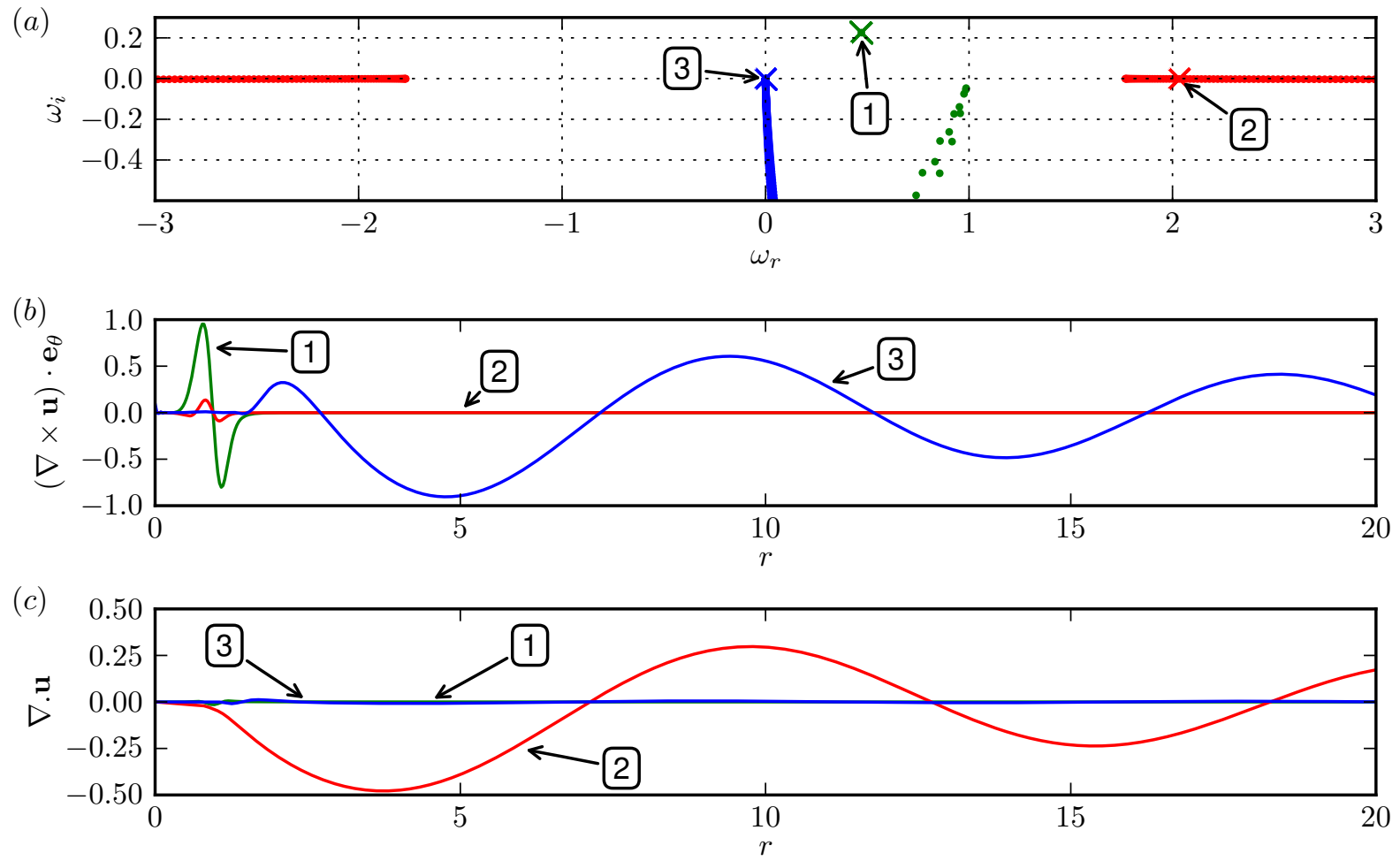

Figure 4: Temporal spectrum directly computed with the QR algorithm for the parallel base flow given by $(15)$ for Re $=500$, $\mathrm{Ma}=0.4$ and $\operatorname{Pr}=1$, and for waves number $k=1$ and $m=1$. (a) The eigenvalue spectrum is displayed in terms of real frequency $\omega_{r}^{\prime}$ and temporal growth rate $\omega_{i}^{\prime}$ of the modes. Blue symbols correspond to vortical modes in the outer flow; red symbols to acoustic modes in the outer flow and green ones to shear-layer modes. (b) The real part of vorticity eigenfunction for three selected modes (indicated by large color crosses in $(a)$ ) (Green: $\omega=0.471+0.226 \mathrm{i}$, label 1 in $(a)$; red: $\omega=2.03-1.91 .10^{-3} \mathrm{i}$, label 2 in $(a)$; Blue: $\omega=2.24 .10^{-6}-1.49 .10^{-3} \mathrm{i}$, label 3 in $\left.(a)\right)$. (c) The real part of dilatation eigenfunction is represented for the same modes as in $(b)$.

with periodic boundary conditions in $x$ and with a streamwise extent $0 \leq x \leq 2 \pi / k$. Using a two-dimensional discretization allows the use of the same code here (for a parallel base flow) and in $\S 4$ (for non-parallel base flow), except for different boundary conditions. The base flow in the present case is still parallel, given by (15). The objective is to compute a number of least stable discrete eigenmodes of the shear-layer type (green symbols in figure $4 a$ ).

If a standard Krylov technique were to be applied to the propagator of the linear equations of motion alone, only the single unstable shear-layer mode could be extracted, all other discrete modes being masked by the less stable acoustic (red in figure 4) or vortical (blue) branches. The SR technique allows to stabilize all modes outside a region of interest of the spectrum, and thereby may give access to otherwise masked parts of the spectrum, in particular here by stabilizing the continuous branches.

The extended linear system (6) is discretized on an orthogonal grid, resolving the domain $0 \leq r \leq 15$, $0 \leq x \leq 2 \pi / k$ with $30 \times 256$ points. Explicit $5^{t h}$-order centered finite differences in combination with a spatial filtering scheme [6] are used for the spatial derivatives. The spatial filter merely suppresses numerical instabilities of the finite-difference scheme; it is unrelated to the temporal filtering employed by the SR method. Matrix-free time stepping of the extended linear equations is performed using a $3^{\text {rd }}$-order RungeKutta algorithm. For the purpose of validation against the results of $\S 3.2$, only modes with a streamwise wavenumber $k=1$ are sought, and higher harmonics of the periodic domain are continuously filtered out during the time stepping, by means of an FFT in $x$. 
Choice of transformation parameters. In order to focus on the green branch represented in figure 4a, appropriate parameter values for $\chi, \tau$ and $\omega_{0}$ must be chosen. As discussed in $\S 2.2$, a value of $\chi^{\prime}=1$ is maintained, such that $\chi=\tau$. The frequency shift $\omega_{0}$ selects the region of interest in the frequency plane; a choice of $\omega_{0}=1+0.2 \mathrm{i}$ has been found to be suitable. The parameter $\tau$ determines the width of the bandpass filter. It should be chosen sufficiently small to efficiently damp undesired modes (here, the blue and red branches) but large enough such that the modes of interest achieve growth rates $\omega_{i} \gtrsim \omega_{0, i}-2 \tau$, as discussed in $\S 2.3$. A value of $\tau=0.5$, yielding $\chi=0.5$, represents a good compromise for the present case. The propagation time is set to $\Delta t=0.5$.

Results. Ten eigenvalues obtained with the SR method are shown as black circles in figure 5, alongside the reference solution (identical to figure $4 a$ ). The agreement is excellent, with relative errors smaller than $10^{-4}$ on both the eigenvalues and the eigenmodes (in $L^{\infty}$ norm). The spectral transformation with the present choice of parameters successfully selects the least stable shear-layer modes. Contour lines in figure 5 indicate the transformed growth rate $\Omega_{i}(\omega)$ that governs the mode selection by the SR method.

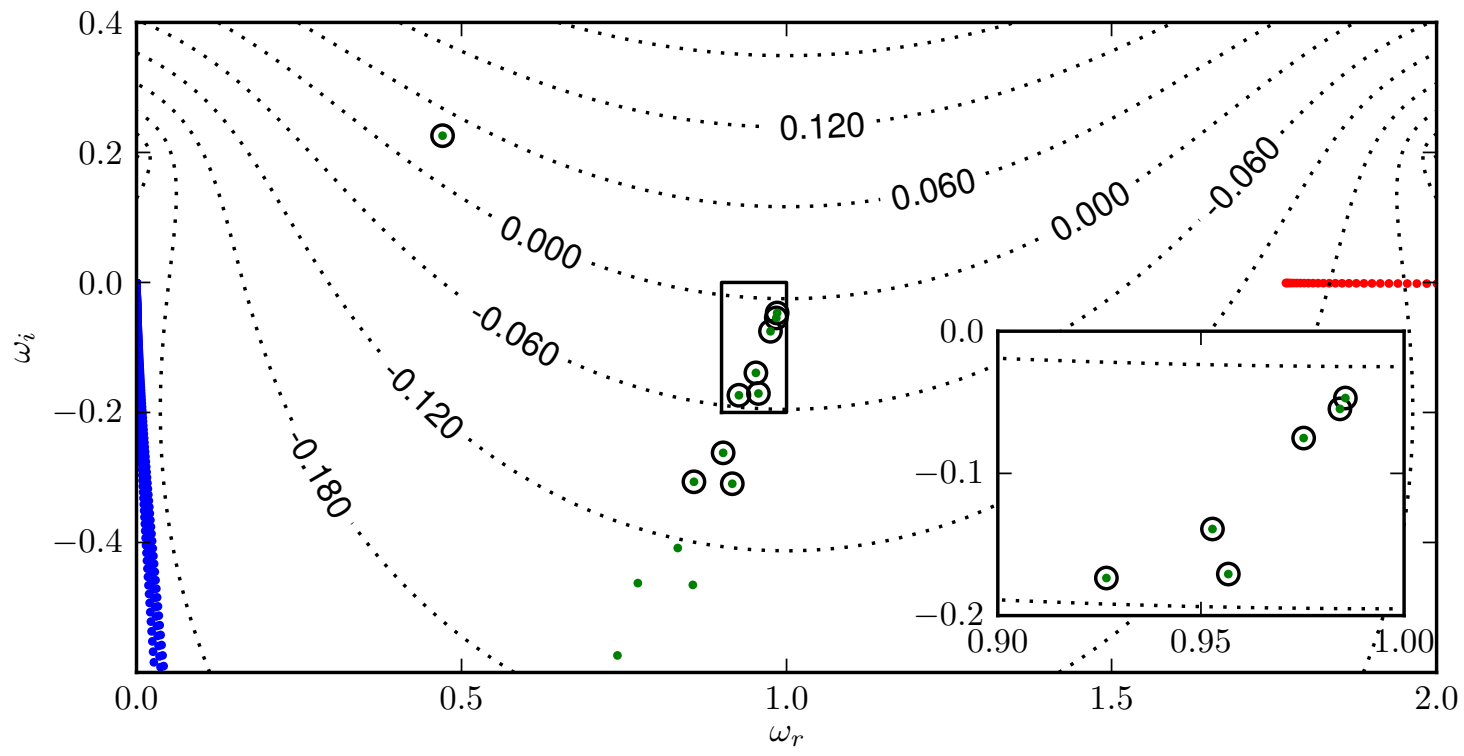

Figure 5: Application of the SR method to the computation of local modes of a compressible jet flow for the same parameter settings as in figure 4 . The spectrum from figure $4 a$ is reproduced with the same conventions. The ten modes computed with the SR method are denoted by black circles. The dotted lines represent isocontours of the growth rate $\Omega_{i}^{+}$of the filtered propagator, based on parameters $\omega_{0}=1+0.2 \mathrm{i}$ and $\tau=\chi=0.5$, confirming that the computed eigenvalues correspond to the largest $\Omega_{i}^{+}$. The insert on the bottom right of the figure shows a close-up view on the region marked by a black rectangle

\section{Application: global eigenmodes of a compressible jet}

\subsection{Non-parallel base flow}

The SR method is now applied to compute global modes (18) of a non-parallel jet. The geometry of the computational domain is represented in figure 6 . The jet exits from an idealized nozzle, modeled as an infinitely thin adiabatic wall at $r=1$ and $x \leq 0$. Only the upper half-plane $r>0$ is resolved in the calculations, and appropriate symmetry conditions for axisymmetric flow are imposed on the jet axis $r=0$. In order to control the jet profile at $x=0$ (diffusive effects inside the pipe can be particularly important at low Reynolds and high Mach numbers), velocity and temperature profiles are imposed at $x=x_{0}$, close to the jet nozzle. The Navier-Stokes equations are solved downstream of $x_{0}$ (domain II in figure $6 a$ ), with local 


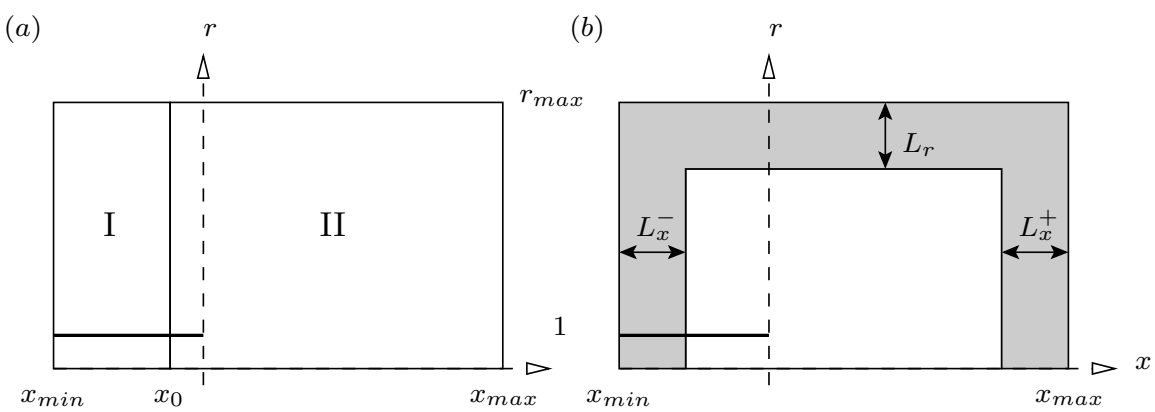

Figure 6: Computational domains for $(a)$ base flow and $(b)$ global mode computations. The parameter values for these computations are: $x_{\min }=-200, x_{\max }=300, r_{\max }=250, x_{0}=-2, L_{x}^{+}=L_{x}^{-}=L_{r}=150$. The base flow is taken to be parallel in region I, whereas the Navier-Stokes equations are solved in region II.

one-dimensional (LODI) boundary conditions [21] on the numerical boundaries at $x_{\min }, x_{\max }$ and $r_{\max }$. The same explicit finite-difference scheme as described in $\S 3.3$ is used. A steady solution of the nonlinear Navier-Stokes equations, computed via the SFD method of [1] for $\operatorname{Re}=100, \mathrm{Ma}=0.75, \operatorname{Pr}=1$ and $S=1$ is taken as a base flow. A parallel flow region is added upstream of $x_{0}$ (domain I in figure $6 a$ ) to extend the base flow to the domain used for the linear stability calculations. Axial velocity and angular vorticity distributions of the spatially spreading base flow are shown in figure $7 a, b$. The momentum thickness

$$
\theta(x)=\int_{0}^{\alpha} \frac{\rho^{b}(r, x) u^{b}(r, x)}{\rho^{b}(0, x) u^{b}(0, x)}\left(1-\frac{u^{b}(r, x)}{u^{b}(0, x)}\right) r \mathrm{~d} r, \quad \alpha=\left\{\begin{array}{cc}
1 & x \leq 0 \\
\infty & x>0
\end{array}\right.
$$

is displayed in figure $7 c$, and selected axial velocity profiles are shown in figure $7 d$.

Temporal eigenmodes of the linearized Navier-Stokes equations, commonly referred to as "global modes", are computed on the domain displayed in figure $6 b$. Non-reflecting boundary conditions given by [7] are employed at the inflow, outflow and upper boundary. Furthermore, perturbation quantities are artificially attenuated in sponge layers [8], indicated by the outer gray regions in figure $6 b$, in order to further minimize spurious reflections. Only axisymmetric modes $(m=0)$ are computed, and symmetry conditions on the jet axis are imposed accordingly. The numerical domain spanned by $-200 \leq x \leq 300$ and $0 \leq r \leq 250$ is discretized with $1024 \times 512$ grid points.

Five eigenvalues were requested for each of nine shift parameters $\omega_{0}$ with $0.3 \leq \omega_{0, r} \leq 0.75$ and $\omega_{0, i}=0.05$, represented by black diamonds in figure 8a. Note that SLEPc may return more than the requested number of eigenvalues. Parameters $\tau=\chi=\Delta t=0.1$ were used in all calculations. The resulting eigenfrequencies are represented in figure $8 a$ in the complex $\omega$ - plane and form a discrete branch of solutions. For each value of $\omega_{0}$, one isocontour of the growth rate $\Omega_{i}^{+}$of the filtered propagator is drawn, corresponding to the growth rate of the most stable mode computed with each particular shift; no eigenfrequency other than those computed should lie above these parabola-shaped curves.

For $\omega_{0}=0.8+0.05 i$, five eigenvalues were first requested, as for the other values of the frequency shift. The computation was then continued to yield a total of fifteen modes. This portion of the spectrum is displayed in more detail in figure $8 c$, together with iso-contours of the growth rate of the filtered propagator. The first five modes that have been found are marked by red circles. Among the additional requested modes, several are found to lie on a separate, slightly more attenuated branch. The spatial structure of the real part of vorticity perturbations associated with three eigenmodes of the upper branch, marked by labels in figure 8 , are displayed in figure 9 . Perturbations are concentrated in the jet shear layer region, suggesting an inflectional instability mechanism. The time evolution of these modes show that the vortical structures represented in figure 9 propagate downstream at a phase velocity of about half the jet centerline velocity; as a consequence, their typical axial wavelength decreases with increasing real frequency. Modes belonging to the more attenuated branch, which is only detected in figure 8 for the shift $\omega_{0}=0.8+0.05 \mathrm{i}$, display a very similar structure (not shown). 
(a)

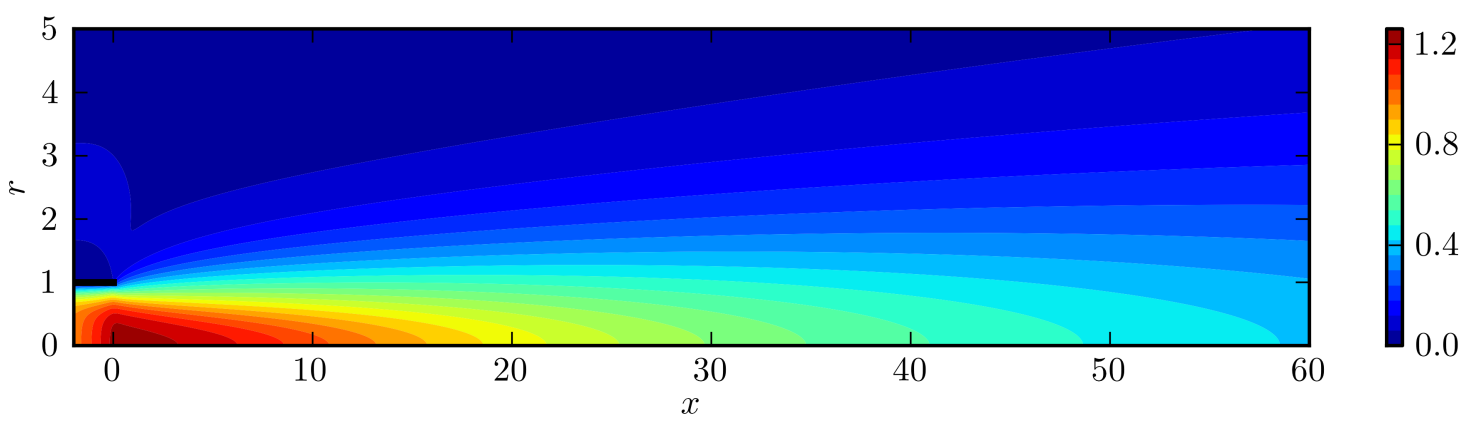

(b)

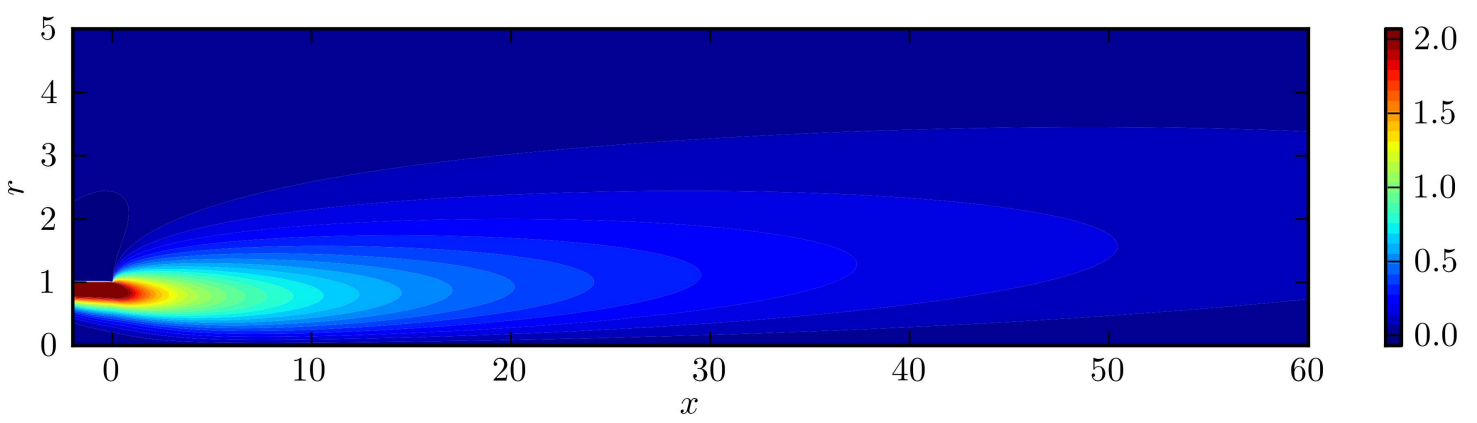

$(c)$

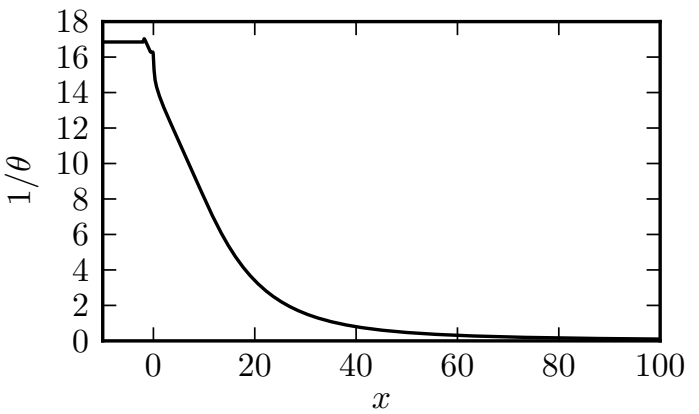

$(d)$

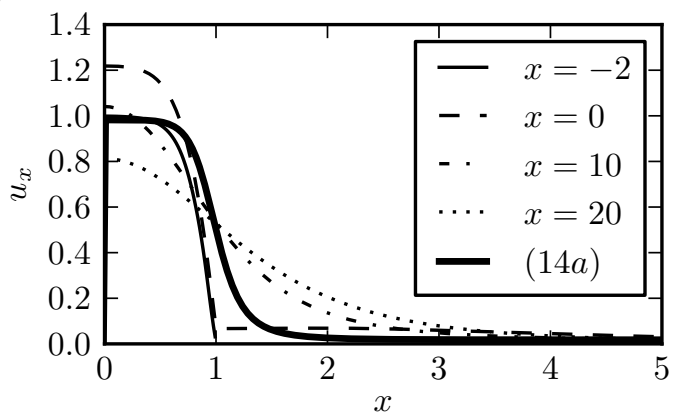

Figure 7: Base flow for the global linear stability study of a spatially developing jet with $\operatorname{Re}=100, \operatorname{Ma}=0.75, \operatorname{Pr}=1$ and $S=1$ (unheated). The axial velocity and angular vorticity distributions are respectively displayed in $(a)$ and $(b)$. Only a portion of the computational domain is shown. The evolution of the momentum thickness $\theta$ is represented in $(c)$. In $(d)$, axial velocity profiles are at various locations are shown together with the profile used in $\S 3.2$ and 3.3 . 
Fifteen modes were computed for a large frequency shift $\omega_{0}=5.1$. These are represented in figure $8 b$ by red and green symbols. Their dilatation fields, shown in figure 10, characterize these modes as being of acoustic nature. Comparing the real and imaginary parts (not shown) of the dilatation fields in the pipe, it appears that some modes correspond to acoustic waves propagating downstream inside the duct and being scattered at the nozzle exit (green symbols in figure 8b). Others correspond to acoustic waves emanating from a location close to the nozzle exit (red symbols in figure $8 b$ ). These modes propagate upstream in the inlet duct.

One should keep in mind that free-stream vortical modes with near zero decay rates are also present in the spectrum, similar as in the local analysis of $\S 3.2$. Without shifting and relaxing of the propagator, all modes shown in figure 8 would be masked by those least stable modes. In practice, however, the computation of these modes is challenging, as the separation between eigenvalues is very weak (on the order of $\left.1 /\left[\operatorname{Re}\left(x_{\max }-x_{\min }\right)\right]\right)$. No result could be converged with sufficient accuracy for this family of modes, and they are not shown.

\subsection{Convergence}

Convergence of the eigenvectors through the iterations of the Krylov-Schur algorithm is usually monitored in terms of a residual associated with the operator the algorithm is applied to, i.e the SR propagator in the present case. Denoting by $\boldsymbol{Q}_{j, p}$ the estimated $p^{t h}$ leading eigenvector after the $j^{\text {th }}$ restart of the Krylov-Schur algorithm, and by $\lambda_{j, p}$ the corresponding eigenvalue, the residual is estimated

$$
e_{j, p}=\frac{\left\|\exp (\Delta t \mathcal{F}) \boldsymbol{Q}_{j, p}-\lambda_{j, p} \boldsymbol{Q}_{j, p}\right\|}{\left\|\boldsymbol{Q}_{j, p}\right\|}
$$

and $\boldsymbol{Q}_{j, p}$ is accepted as converged if the estimation is smaller than a user-chosen tolerance $\epsilon$. A typical evolution of the estimation of this residual is displayed in figure 11a. Non-monotonous convergence is observed, consistent with the fact that, in the present case for which the operator $L$ is non-Hermitian, the filtered propagator is non-Hermitian as well. For $\omega_{0}=0.45+0.05 \mathrm{i}$, computations were performed with values $\epsilon=10^{-3}$ ( $\times$ in figure $\left.11 b\right), 10^{-4}(\circ)$ and $10^{-5}(+)$. Five modes were requested in each computation. The resulting spectra are shown in figure 11b. Significant scattering of the computed eigenvalues is noticed for $\epsilon=10^{-3}$. The absolute scattering being of the same order of magnitude in the real and imaginary directions, relative errors in the real part of the eigenfrequency are much smaller than in the growth rate. The scattering of eigenvalues becomes less important as requested accuracy increases. With $\epsilon=10^{-4}$ and $\epsilon=10^{-5}$ eigenvalues seem to converge to a line trend, and eigenvalues in the overlapping region from the two shift values $\omega_{0}=0.45+0.05 \mathrm{i}$ and $0.5+0.05 \mathrm{i}$ are in reasonably good agreement, as shown in figure $11 c$. However, this measure of convergence depends on the spectral transformation used, in particular here on $\Delta t$ and $\tau$. A more meaningful measure of accuracy may be defined based on the original operator $L$ :

$$
e_{j, p}^{\prime}=\frac{\left\|L \boldsymbol{q}_{j, p}+\mathrm{i} \omega_{j, p} \boldsymbol{q}_{j, p}\right\|}{\left\|\boldsymbol{q}_{j, p}\right\|} \quad \text { with } \boldsymbol{Q}_{j, p}=\left(\boldsymbol{q}_{j, p}, \overline{\boldsymbol{q}}_{j, p}\right)^{T}
$$

These residuals are computed after convergence (denoted by $j=\infty$ ), and are displayed in figure $11 d$. A first observation is that the values differ significantly from the values of the $e_{\infty, p} \approx \epsilon$ discussed earlier, measured with respect to the SR propagator. Furthermore, the values of $e_{\infty, p}^{\prime}$ decrease only slowly with $\epsilon$; values on the order of $3 \%, 2 \%$ and $1 \%$ are found for $\epsilon=10^{-3}, 10^{-4}$ and $10^{-5}$ respectively. This saturation seems to be due to the spatial filtering which is applied during time-stepping in order to maintain stability. It introduces a slight modification of the propagator which is not taken into account when the Rayleigh quotient (12) is taken.

In order to achieve residuals $e^{\prime} \leq 2.2 \%$, values of $\epsilon$ ranging from $10^{-4}$ to $10^{-7}$ had to be used for the computations presented in the previous section, depending on the shift $\omega_{0}$ (larger values of $\omega_{0, r}$ requiring smaller values of $\epsilon$ in the present case). 


\section{Conclusion}

A new numerical procedure for the solution of large eigenvalue problems has been presented. A relaxation technique using a first-order temporal bandpass filter is coupled to to the linearized equations of motion, such that the least stable eigenmodes of the filtered system lie in a prescribed frequency band of interest centered around a shift frequency. These modes are then recovered through propagation over a finite time interval, using standard eigenvalue extraction techniques. This "shift-relax" transformation therefore requires no solution of linear systems, which are computationally expensive or even untractable for global stability problems involving two- or three-dimensional flows. Although not as flexible as the classical "shift-invert" transformation or its variants, the present method has the advantage of considerably lower memory requirement, making it suitable for the analysis of complex two- or three-dimensional flow geometries. Another advantage lies in the ease of implementation: only a simple filter equation needs to be added to an existing simulation code in order to perform eigenmode extraction. No matrix needs to be built, and no preconditioning is required. Finally, the algorithm can be parallelized as efficiently as a regular time stepper, as the filter is local in space.

\section{Acknowledgments}

This work was supported by DGA grant number 2009.60.034.00.470.75.01. The authors are thankful to Miguel Fosas and Patrick Huerre for their comments. 
(a)

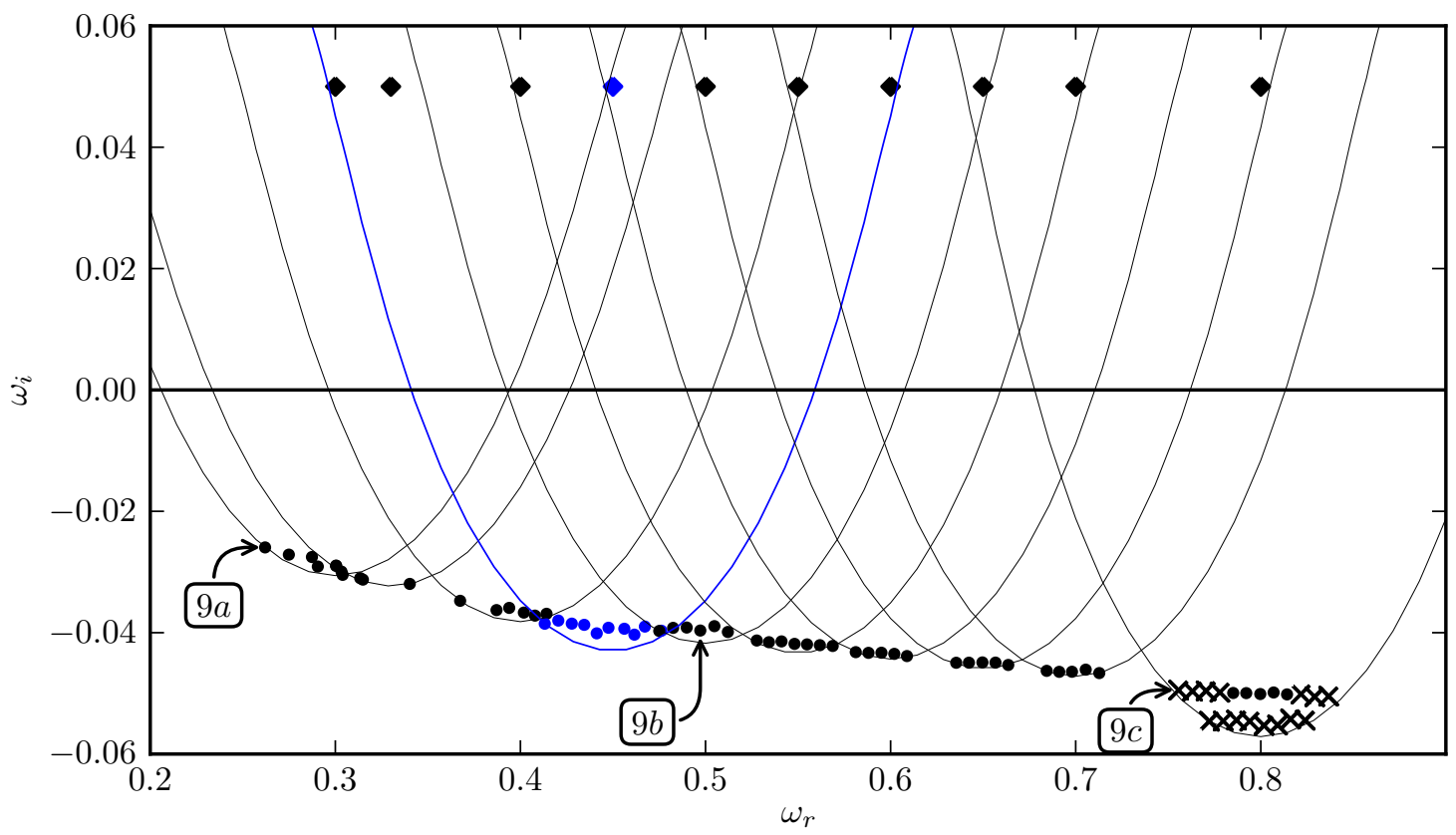

(b)

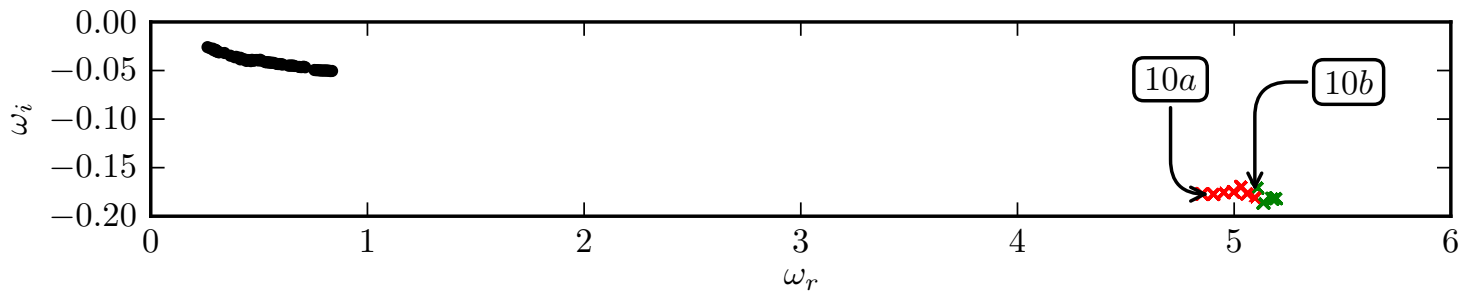

(c)

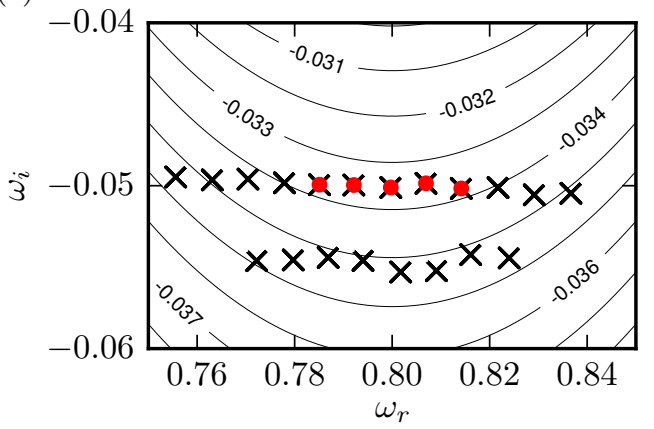

Figure 8: Eigenvalues of axisymmetric modes in an isothermal jet at $\mathrm{Re}=100, \mathrm{Ma}=0.75$ and $\operatorname{Pr}=1$ computed using the SR method. Tolerance was set to $\epsilon=10^{-4}$ for $\omega_{0, r} \leq 0.5, \epsilon=10^{-5}$ for $0.5<\omega_{0, r} \leq 0.8$ and $\epsilon=10^{-7}$ for $\omega_{0, r}=5.1$. (a) Low frequency shear layer modes. Shift positions are indicated by diamonds. For each shift, an isocontour of the filtered propagator growth rate is represented; its value corresponds to the least amplified mode computed by the Krylov-Schur solver. For $\omega_{0}=0.45+0.05 i$, the shift, the modes and the isocontour are represented in blue. (b) Low frequency vortical modes are shown together with higher frequency acoustic modes. (c) Close-up on the 15 modes computed for $\omega_{0}=0.8+0.05 \mathrm{i}$, showing the existence of two branches: dots correspond to the first five modes computed, crosses correspond to the next ten. Labels correspond to the modes for which the vorticity or dilatation field is displayed in figure 9 and 10 . 

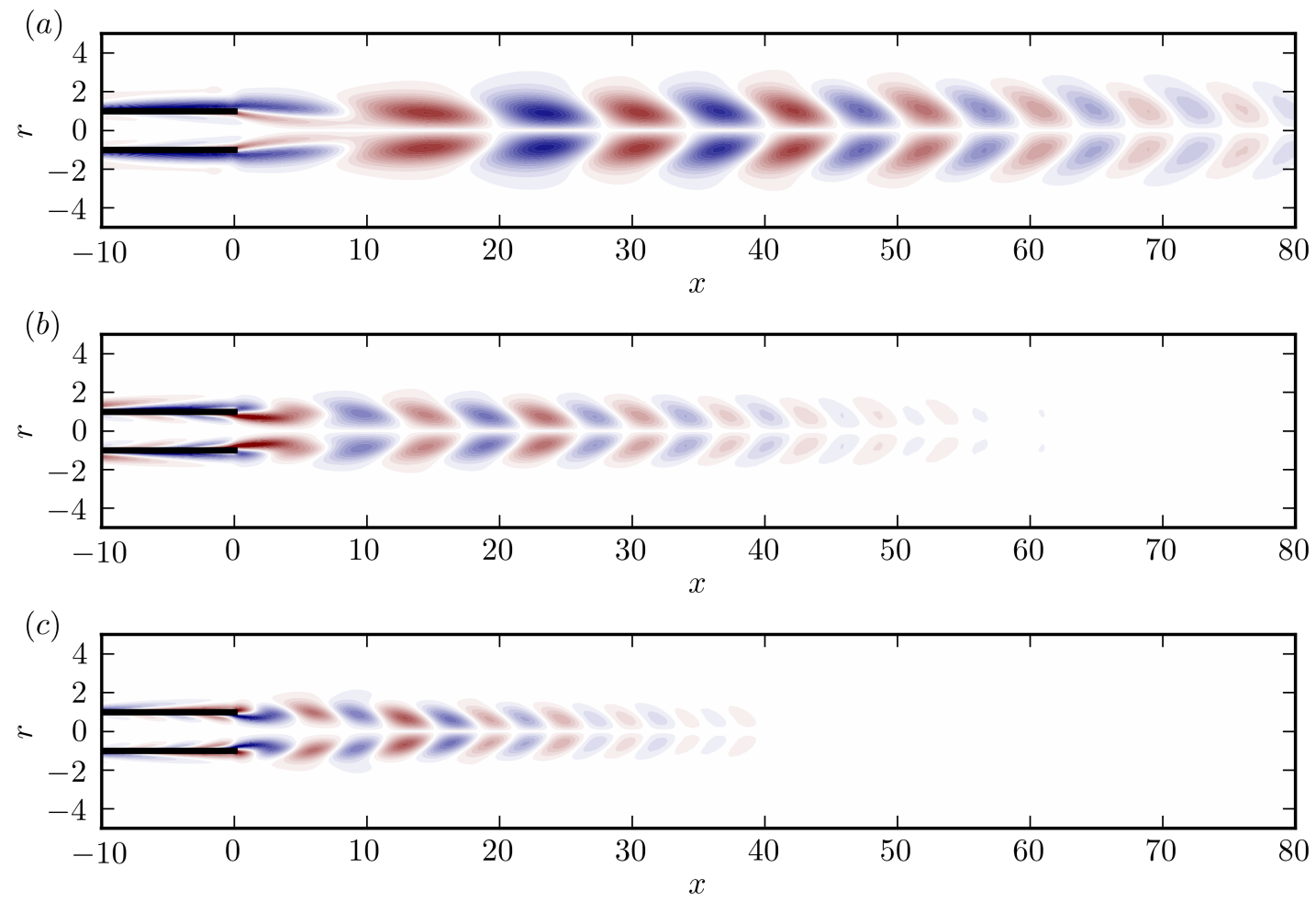

Figure 9: Real part of the vorticity fields of typical eigenmodes, labeled in figure $8 a$, in an isothermal jet at $\operatorname{Re}=100$, Ma $=0.75$ and $\operatorname{Pr}=1$. (a) $\omega=0.26-0.026 \mathrm{i}$. (b) $\omega=0.50-0.040 \mathrm{i}$. (c) $\omega=0.71-0.047 \mathrm{i}$.
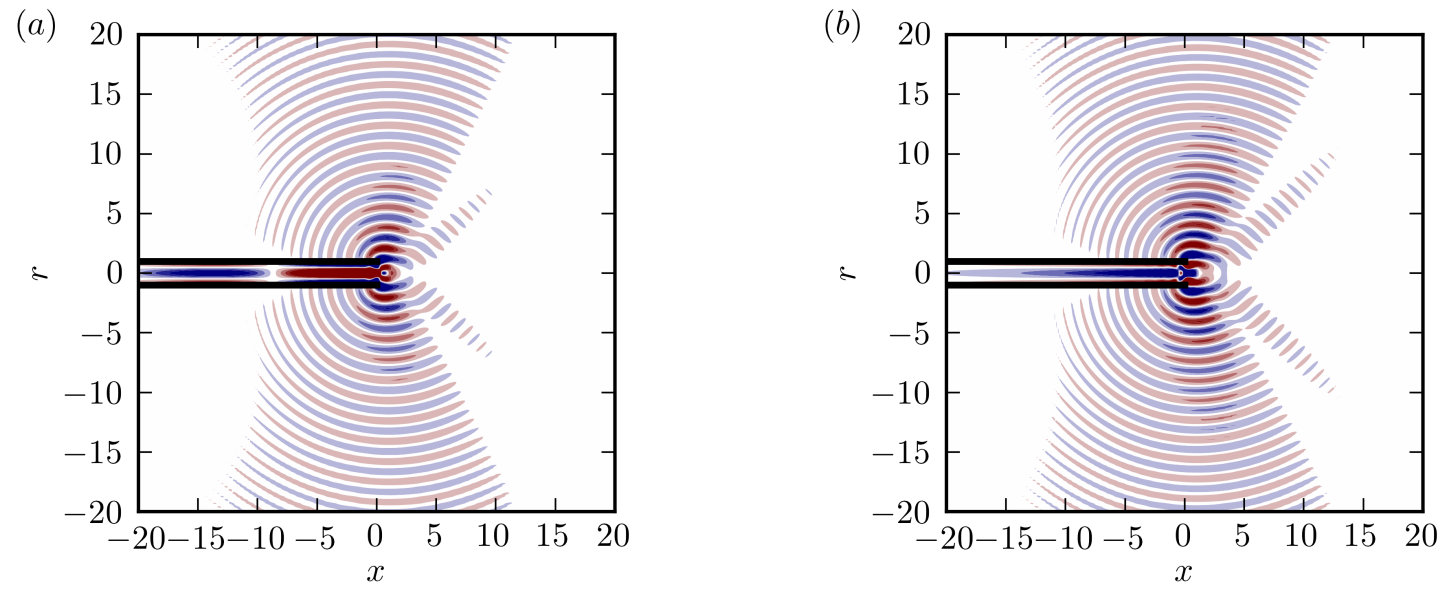

Figure 10: Real part of the dilatation fields of typical eigenmodes, labeled in figure $8 a$, in an isothermal jet at Re $=100$, $\mathrm{Ma}=0.75$ and $\operatorname{Pr}=1$. (a) $\omega=4.9-0.17 \mathrm{i}$. (b) $\omega=5.1-0.18 \mathrm{i}$. 
(a)

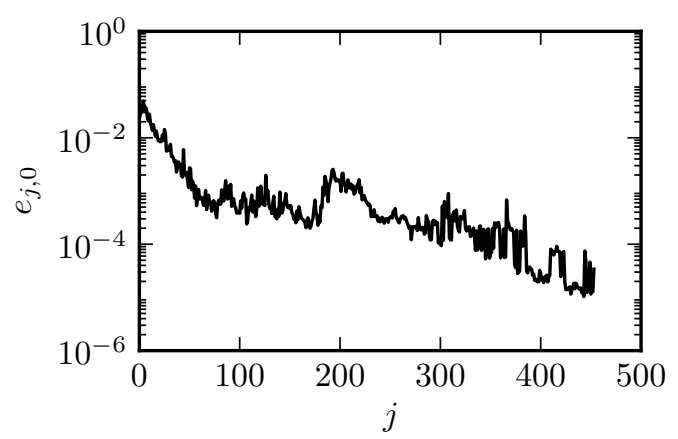

(c)

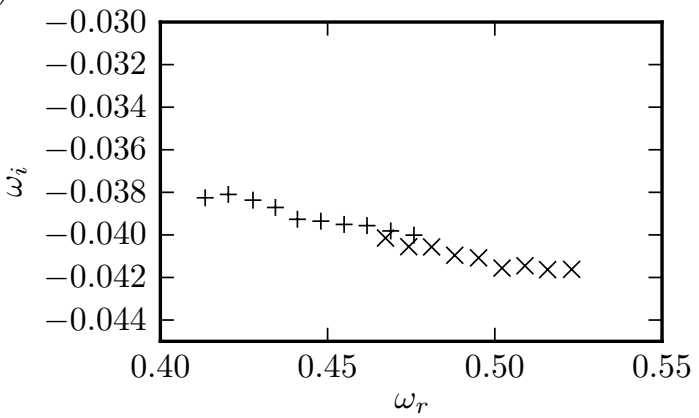

$(b)$

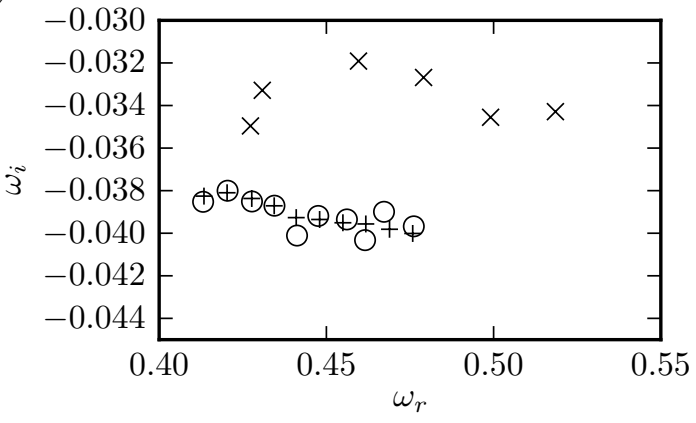

$(d)$

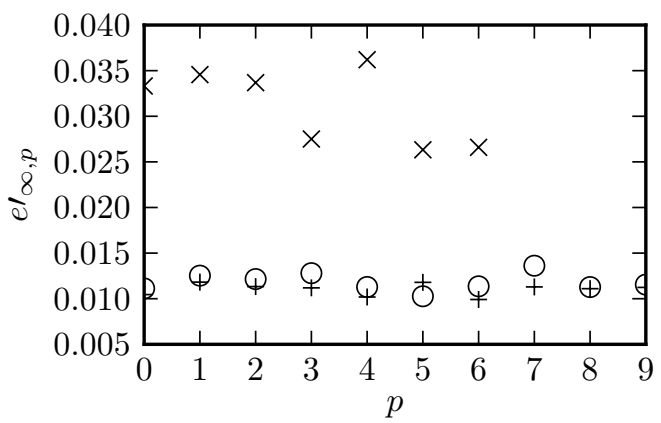

Figure 11: Convergence features. (a) A typical evolution of the estimated residual of the leading eigenvalue with the number of restarts. (b) Spectra computed with $\omega_{0}=0.45+0.05 i$ and $\epsilon=10^{-3}, 10^{-4}$ and $10^{-5}$ (respectively represented by $\times, \circ$ and + symbols). 5 modes were requested. (c) Spectra computed for $\epsilon=10^{-5}$ with $\omega_{0}=0.45+0.05 \mathrm{i}$ and $0.5+0.05 \mathrm{i}$ are shown. $(d)$ Residual based for the computations of $(b)$. 


\section{Appendix A. Distinction between the two transformed eigenvalues}

The eigenvalue transformation is given in terms of the scaled variables by (10). Let us introduce

$$
\widetilde{\Omega}=\frac{\Omega^{\prime}+\mathrm{i}}{\sqrt{\chi^{\prime}}}, \quad \widetilde{\omega}=\frac{\omega^{\prime}+\mathrm{i}\left(1-\chi^{\prime}\right)}{\sqrt{\chi^{\prime}}} .
$$

Equation (10) then reads

$$
\widetilde{\Omega}^{2}-\widetilde{\omega} \widetilde{\Omega}+1=0 .
$$

The solutions of this second-order polynomial are given by

$$
\widetilde{\Omega}=\frac{1}{2}\left(\widetilde{\omega} \pm \sqrt{\widetilde{\omega}^{2}-4}\right) .
$$

Let $\alpha=\sqrt{\widetilde{\omega}^{2}-4}$. One of these roots has a positive imaginary part and the other one a negative one if $\left|\widetilde{\omega}_{i}\right| \leq\left|\alpha_{i}\right|$. In order to prove that this condition holds, real and imaginary parts are introduced in the definition of $\alpha$ :

$$
\left(\alpha_{r}^{2}-\alpha_{i}^{2}\right)+2 \alpha_{r} \alpha_{i} \mathrm{i}=\left(\widetilde{\omega}_{r}^{2}-\widetilde{\omega}_{i}^{2}\right)-4+2 \widetilde{\omega}_{r} \widetilde{\omega}_{i} \mathrm{i}
$$

so

$$
\widetilde{\omega}_{r} \widetilde{\omega}_{i}=\alpha_{r} \alpha_{i} \quad \alpha_{r}^{2}-\alpha_{i}^{2}=\widetilde{\omega}_{r}^{2}-\widetilde{\omega}_{i}^{2}-4 .
$$

The variable $\alpha_{i}$ can then be written as

$$
\alpha_{i}^{2}=\widetilde{\omega}_{i}^{2}+4-\left(\widetilde{\omega}_{r}^{2}-\alpha_{r}^{2}\right)=\widetilde{\omega}_{i}^{2}+4-\left(\frac{\alpha_{i}^{2}}{\widetilde{\omega}_{i}^{2}}-1\right) \alpha_{r}^{2}
$$

which finally gives

$$
\alpha_{i}^{2}=\frac{\widetilde{\omega}_{i}^{2}+4+\alpha_{r}^{2}}{1+\frac{\alpha_{r}^{2}}{\widetilde{\omega}_{i}^{2}}}=\widetilde{\omega}_{i}^{2} \frac{\widetilde{\omega}_{i}^{2}+4+\alpha_{r}^{2}}{\widetilde{\omega}_{i}^{2}+\alpha_{r}^{2}} \geq \widetilde{\omega}_{i}^{2} .
$$

Let $\widetilde{\Omega}^{+}$denote the root of (A.1) with a positive imaginary part and $\widetilde{\Omega}^{-}$the one with the negative imaginary part. In terms of the primed variables, this corresponds to

$$
\Omega_{i}^{\prime-} \leq-1 \leq \Omega_{i}^{\prime+}
$$

\section{References}

[1] E. Akervik, L. Brandt, D.S. Henningson, J. Hoepffner, O. Marxen, and P. Schlatter. Steady solutions of the Navier-Stokes equations by selective frequency damping. Phys. Fluids, 18(6):068102, 2006.

[2] E. Akervik, U. Ehrenstein, F. Gallaire, and D.S. Henningson. Global two-dimensional stability measures of the flat plate boundary-layer flow. Eur. J. Mech. B-Fluid, 27(5):501-513, 2008.

[3] P.R. Amestoy, I.S. Duff, and J.Y. L'Excellent. Multifrontal parallel distributed symmetric and unsymmetric solvers. Comput. Method. Appl. M., 184(2-4):501-520, 2000.

[4] S. Balay, K. Buschelman, V. Eijkhout, W.D. Gropp, D. Kaushik, M.G. Knepley, L. Curfman McInnes, B.F. Smith, and H. Zhang. PETSc users manual. Technical Report ANL-95/11 - Revision 3.0.0, Argonne National Laboratory, 2008. Available at http://www.mcs.anl.gov/petsc/petsc-as/.

[5] A. Barbagallo, D. Sipp, and P.J. Schmid. Closed-loop control of an open cavity flow using reduced-order models. J. Fluid Mech., 641:1-50, 2009. 
[6] J. Berland, C. Bogey, O. Marsden, and C. Bailly. High-order, low dispersive and low dissipative explicit schemes for multiple-scale and boundary problems. J. Comput. Phys., 224(2):637-662, 2007.

[7] C. Bogey and C. Bailly. Three-dimensional non-reflective boundary conditions for acoustic simulations: far field formulation and validation test cases. Acta Acust., 88:463-471, 2002.

[8] T. Colonius. Modeling artificial boundary conditions for compressible flow. Annu. Rev. Fluid Mech., 36(1):315-345, 2004.

[9] T.A. Davis. UMFPACK version 5.5.0 user guide. Technical report, 2009. Available at http://www.cise.ufl.edu/research/sparse/umfpack/.

[10] W.S. Edwards, L.S. Tuckerman, R.A. Friesner, and D.C. Sorensen. Krylov methods for the incompressible Navier-Stokes equations. J. Comput. Phys., 110(1):82-102, 1994.

[11] G.H. Golub and C.F. Van Loan. Matrix computations, third edition. The John Hopkins University Press, 1996.

[12] V. Hernandez, J.E. Roman, A. Tomas, and V. Vidal. A survey of software for sparse eigenvalue problems. Technical Report STR-6, Universidad Politcnica de Valencia, 2006. Available at http://www.grycap.upv.es/slepc.

[13] P. Huerre and P.A. Monkewitz. Local and global instabilities in spatially developing flows. Annu. Rev. Fluid Mech., 22:473-537, 1990.

[14] D. A. Knoll and D. E. Keyes. Jacobian-free Newton-Krylov methods: a survey of approaches and applications. J. Comput. Phys., 193(2):357-397, January 2004.

[15] R.B. Lehoucq and A.G. Salinger. Large-Scale eigenvalue calculations for stability analysis of steady flows on massively parallel computers. Inter. J. Numer. Meth. Fl., 36:309-327, 1999.

[16] R.B. Lehoucq, D.C. Sorensen, and C. Yang. ARPACK Users' Guide: Solution of Large-Scale Eigenvalue Problems with Implicitly Restarted Arnoldi Methods. SIAM, 1998.

[17] S.K. Lele. Compact finite difference schemes with spectral-like resolution. J. Comput. Phys., 103(1):1642, 1992 .

[18] C.J. Mack and P.J. Schmid. A preconditioned krylov technique for global hydrodynamic stability analysis of large-scale compressible flows. J. Comput. Phys., 229(3):541-560, 2010.

[19] C.J. Mack, P.J. Schmid, and J.L. Sesterhenn. Global stability of swept flow around a parabolic body: connecting attachment-line and crossflow modes. J. Fluid Mech., 611:205-214, 2008.

[20] J.W. Nichols and S.K. Lele. Global modes and transient response of a cold supersonic jet. J. Fluid Mech., 669:225-241, January 2011.

[21] T.J. Poinsot and S.K. Lele. Boundary conditions for direct simulations of compressible viscous flows. J. Comput. Phys., 101(1):104-129, 1992.

[22] J.E. Roman, M. Kammerer, F. Merz, and F. Jenko. Fast eigenvalue calculations in a massively parallel plasma turbulence code. Parallel Comput., 36(5-6):339-358, 2010. Parallel Matrix Algorithms and Applications.

[23] J.E. Roman, E. Romero, and A. Tomas. SLEPc users manual. Technical Report DSIC-II/24/02, Universidad Politecnica de Valencia, 2010. Available at http://www.grycap.upv.es/slepc.

[24] Y. Saad. Iterative methods for sparse linear systems, second edition. SIAM, 2003. 
[25] R.D. Sandberg. Governing equations for a new compressible Navier-Stokes solver in general cylindrical coordinates. Technical Report AFM-07/07, University of Southampton, 2007. Available at http://eprints.soton.ac.uk/49523/.

[26] P.J. Schmid and D.S. Henningson. Stability and Transition in Shear Flows. Springer, 2001. 